CONNECTIONS BETWEEN MOLECULAR PHOTOIONIZATION AND

ELECTRON-MOLECULE SCATTERING WITH EMPHASIS ON SHAPE RESONANCES

by

J.L. Dehmer and Dan Dill

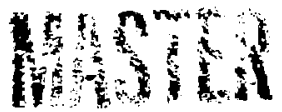

Prepared for

Symposium on

Electron-Molecule Collisions Satellite Meeting

XI ICPEAC

Tokyo, Japan

September $6-7,1979$

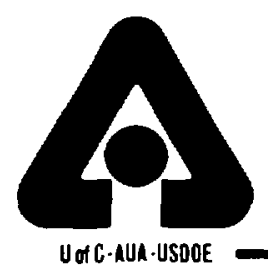

ARGONNE NATIONAL LABORATORY, ARGONNE, ILLINOIS

Operated under Contract W-31-109-Eng-38 for the

U. S. DEPARTMENT OF ENERGY 


\title{
CONNECTIONS BETWEEN MOLECULAR PHOTOIONIZATION AND ELECTRON-MOLECULE SCATTERING WITH EMPHASIS ON SHAPE RESONANCES*
}

\author{
J. L. Dehmer
}

Argonne National Laboratory, Argonne, Illinois 60439 U.S.A.

and

Dan Dill

Department of Chemistry, Boston University, Boston, Massachusetts 02215 U.S.A.

Most of our detailed information on the spectroscopy and dynamics of the electronic continuum of molecules is based on the complementary probesphotoionization and electron scattering. Though usually studied separately, it is most useful to appreciate the connections between these two processes since our understanding of one is often the key to interpreting or even generating new results in the other. We approach this subject in two steps. First, we very briefly outline the well-established connections, e.g. , the Bethe-Born theory and comparisons of isoelectronic systems. Then we focus on a point of contact-the role of shape resonances in molecular photoionization and electron-molecule scattering-for which a substantial amount of new information has become available. Specific topics include mapping of resonances from the neutral ( $h \nu+$ molecule) to the negative ion (e+molecule) system, angular distributions, and interaction with vibration.

\section{INTRODUCTION}

For the most part, electron scattering and photoionization are rather different probes of the electronic continuum of molecules: The basic interactions are different. Hence, they access different manifolds of states. All of the energy of the photon is absorbed in a single step, whereas the electron can impart any fraction of its energy. In practice, collision and excitation energies are more easily tuned in scattering experiments, while resolution is generally much better in photon experiments. A greater variety of measurements, e.g., elastic, total, differential, multipiy differential, are possible in scattering experiments. These and other differences between the more rich and varied collision studies and the "cleaner" photon studies provide us with alternative, though often unconnected, views of the dynamics of the molecular continuum. At the same time, there are important points of contact between electron scattering and photoionization which permit us to check results of one against the other, and to transfer spectroscopic and dynamical information back and forth. It is therefore necessary to develop and utilize such connections in order to fully exploit the diversity of electron- and photon-molecule collisions. In this article we briefly review some of the well-

$+$

Work performed under the auspices of the U.S. Department of Energy and National Science Foundation Grant CHE78-08707. Acknowledgement is also made to the Donors of the Petroleum Research Fund, administered by the American Chemical Society, for partial support of this work. 
known connections for the purposes of perspective. The main objective, however, is to draw attention to an emerging framework for comparing electron-molecule scattering $(e+M)$ and molecular photoionization $(h \nu+M)$ on the basis of prominent shape resonances. Note that although these systems have different electron numbers, the short-range nature of these resonances preserves a similarity which persists, though modified, even when the long-range part of the potential is drastically altered.

\section{PSEUDOPHOTONS}

Under certain conditions, inelastic electron collisions simulate photoionization. This is the best known and most often exploited connection between the two processes. Reviewing briefly, we follow Bethe 1,2 in treating the action of a fast electron as a weak perturbation, i.e., within the first Bom approximation. Numerous results thus obtained are powerful as a framework of general understanding of charged-particle collisions with atoms and molecules, as fully discussed in Refs. 3-5. The Bethe formula for the differential cross section is

$$
\frac{d \sigma(K)}{d E}=\frac{4 \pi a_{0}^{2}}{T / R} \cdot \frac{R}{E} \cdot \frac{d f(K)}{d E} \cdot d\left[\ln \left(K a_{0}\right)^{2}\right]
$$

where $\mathrm{T}$ is the incident kinetic energy, $\mathrm{E}$ is the excitation energy, $\hbar \mathrm{K}$ is the momentum transfer, and $\mathrm{df}(\mathrm{K}) / \mathrm{dE}$ is the differential generalized oscillator strength defined by

$$
\frac{\mathrm{d} f(\mathrm{~K})}{\mathrm{dE}}=\left(\frac{E}{R}\right)\left(\mathrm{Ka}_{0}\right)^{-2}\left|\left(E\left|\sum_{j} \exp \left(i \overrightarrow{\mathrm{K}} \cdot \overrightarrow{r_{j}}\right)\right| 0\right)\right|^{2}
$$

Thus, the analysis of fast collisions reduces to the study of an atomic matrix element, just as the analysis of the photoeffect does. The similarity to the photoeffect is not only formal but deep-rocted in the physics. When the incident electron is scattered by a small angle, the momentum transfer $\hbar \mathrm{K}$ is small. Intuitively such a collision may be characterized by a large impact parameter $b \approx 1 / K$. The force then exerted by the incident electron is uniform over the spatial extent of the atom, in the same way as implied by the dipole approximation for photoabsorption. Yet the same force is sharply peaked in time, owing to the high velocity of the incident electron, and therefore its Fourier components are widely and smoothly distributed in frequency $\omega$. Each of the Fourier components excites the atomic state at $E=\hbar \omega$. Consequently, electron collisions at small $\mathrm{K}$ are similar to irradiation with a photon flux having a wide and calculable spectrum, hence the name "pseudophoton."

To see this connection mathematically, one may expand the exponential in $E q .2$ and write the small-K behavior of $\mathrm{df}(\mathrm{K}) / \mathrm{dE}$ as

$$
\frac{d f(K)}{d E}=\frac{d f}{d E}+\left(K a_{0}\right)^{2} \frac{d f^{(1)}}{d E}+\frac{1}{2}\left(K a_{0}\right)^{4} \frac{d f^{(2)}}{d E}+\ldots
$$

where $d f / d E$ is the dipole differential oscillator strength and $d f / d E{ }^{(1)}$ and $\mathrm{df} / \mathrm{dE}{ }^{(2)}$ are defined in Ref. 3. Thus, in principle, we expect to see in electron collisions in the limit of small $\mathrm{K}$ precisely the same dynamics as we see in photoionization.

There are some differences between electron collisions and photoabsorption, however, First of all, the limit $\mathrm{K} \rightarrow 0$ is never precisely realized in any inelastic 
electron collision. The smallest $\mathrm{K}$ value for fixed $\mathrm{E}$ and $\mathrm{T}$ occurs at zero scattering angle, and is given by

$$
\left(\mathrm{K}_{\min } \mathrm{a}_{0}\right)^{2} \cong \mathrm{E}^{2} /(4 \mathrm{RT})
$$

where $E / T \ll 1$. The value of $\left(K_{\left.\min _{0}\right)^{2}}\right.$ is decisive for consideration of the nondipole contributions expressed by the second and higher terms in Eq. 3. Second, by studying the $\mathrm{K}$ dependence of $\mathrm{df}(\mathrm{K}) / \mathrm{dE}$, one can leam much about nondipole excitations, which are often quite sensitive to electron correlations.

A substantial body of work is based on this thesis, and several reviews exist (see, e.g., Refs. 3-7). With regard to the study of the molecular continuum, the work by van der Wiel, Brion, and co-workers (see, e.g., Refs. 7-11) has been most influential. By measuring ejected electrons, photons, and fragment ions in coincidence with the energy lost by forward scattered electrons (the pseudophoton monochromator), these authors have provided a wide variety of simulated photoionization studies, including total and partial photoionization cross sections, photoelectron angular distributions, and photoionization mass spectrometry. Of course, energy-loss spectroscopy in or near the dipole region has been used extensively in the study of molecular pseudo-optical spectroscopy, and recent work by Read and co-workers (see, e.g., Refs. 12 and 13, and F. Read, this volume) has achieved very high resolution in inner-shell excitation (equivalent to $0.01 \AA$ at $43 \AA$ ) that photon measurements will be hard pressed to equal. Work in the other direction across this interface has been performed by Kim, 14 who has utilized photoabsorption data to simulate the soit collision part of the secondary electron cross section for electron (and heavy ion) collisions with atoms and molecules.

\section{ISOELECTRONIC SYSTEMS}

At lower incident energies, the electron can no longer be treated as an external agent, acting as a weak perturbatior rather, its (penetrating) low- $\ell$ components become incorporated in the collision complex and must be treated on an equal footing with the other electrons. The criterion for this transformation is whether the incident electron is fast or slow compared to the internal motion of target electrons involved in the phenomenon of interest. For slow electron collisions, therefore, the point of contact between electron scattering and photoionization is the isoelectronic system. Hence, $h v+M$ is to be compared with $e+M^{+}$, $\mathrm{h} v+\mathrm{M}^{-}$with $\mathrm{e}+\mathrm{M}$, and so on. In this way it should be possible to create the same collision complex using either electrons or photons, although access to particular states of this complex will be govemed by selection rules. The strategy of studying the same state by means of altemative probes has obvious advantages. The spectroscopy and dynamical aspects of the collision complex are the same, thus showcasing the differences due to details of the excitation mechanism.

The main effort toward providing a unifled framework for treating continuous optical spectra and electron collisions has been led by Fano, Seaton, and coworkers. In that work, the properties of entire spectra-specifically the line positions and transition probabilities of perturbed series and of their adjoining continua as well as autoionization line profiles and related collision cross sections - have been interpreted in terms of a few parameters, such as quantum defects and oscillator-strength densities, which vary slowly with the excitation energy. Multichannel quantum defect theory (MQDT) and frame transformation procedures are the foundations of this work. The reader is referred to Ref, 15 for a summary of the concepts and procedures employed for these purposes since 1969 . 
There, reference is also made to the treatment of negative ions and to the direct $a b$ initio calculation of the fundamental MQDT parameters and their comparison with experiment. Though developed mainly in the context of atomic physics, applications to molecules (see, e.g., Refs. 15-17) have proven extremely suc cessful, indicating a major impact on molecular systems in the future.

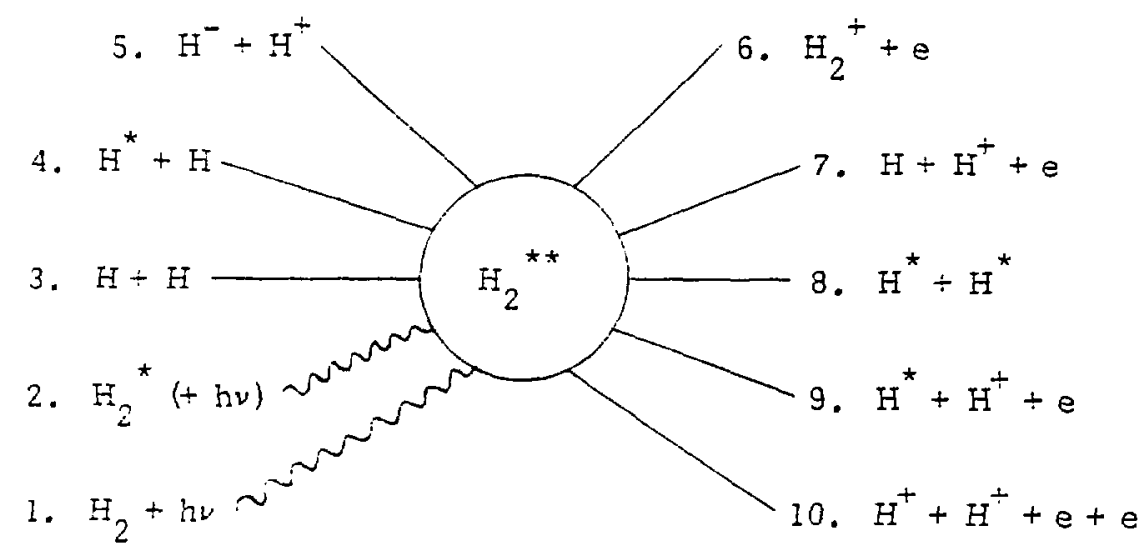

FIG. 1.--Altemative observation channels for the doubly excited $\mathrm{H}_{2}{ }^{*}$ complex.

Actually, the connection between electron scattering and photoionization is only part of a much larger picture, as represented by an example taken from Ref. 18. In Fig. 1 ten channels are indicated which can, in principle, lead to the same complex represented here by a doubly excited hydrogen molecule. Hence, certain states of a particular system can be viewed as a point of contact between diverse isoelectronic collision channels, involving heavy particles in addition to electrons and photons. Altematively, this manifold of collision channels can be viewed as a means of detecting the products of photoionization and electron collisions with ground state $\mathrm{H}_{2}$. Note that Fig. 1 suppresses vibrational and rotational degrees of freedom for the sake of brevity. Actually, these modes are central to the study of the molecular continuum, and, together with molecular symmetry, account for the great variety of physical phenomena in molecular physics relative to atomic physics. An example of the role of vibrational motion is the modification of the net transition probabilities to a single collision complex which can be caused by Franck-Condon factors associated with altemative initial states. Another is vibrational autoionization in which vibronic coupling transfers vibrational energy into electron energy, with the expulsion of a continuum electron.

The main difficulty with this dual approach to the study of collision dynamics is that one of the entrance channels always involves molecular ions which are difficult to produce in abundance in the laboratory. Therefore, whereas electronmolecule collisions have been studied extensively (see, e.g., Ref. 12) for many molecules over broad energy ranges, and for many exit channels, photoionization of negative ions has been largely confined to narrow-energy ranges by the need to use laser excitation to compensate for low number densities. This does not diminish the significance of the photodetachment work (see e.g., Refs. 20-26) which has led to the determination of electron affinities and near-threshold behavior for several negative molecular ions. However, the scope of comparison with electronmolecule scattering is severely limited. A recent advance, 27 which may significantly abate the wavelength limitations of laser photodetachment, involves utilizing fast ion beams to Doppler shift laser light so as to increase the accessible tunable range. In an experiment on $\mathrm{h} v+\mathrm{H}^{-}, 1 \mathrm{P}$ Feshbach and shape resonances $\sim 11 \mathrm{eV}$ above the ground state of $\mathrm{H}$ were observed at high resolution. Comparison with theory $28-30$ was very good, as was the analogous comparis on with $\mathrm{e}+\mathrm{H}$ 
data. 31 This type of study in molecules would be a complementary check of electron-molecule data and would result in more detailed study of vibrational (and also perhaps rotational) structure of high-energy negative ion molecular states.

The situation is similar for the neutral system, where a great body of data exists for molecular photoionization, while a much smaller, though expanding, body of data exists for electron-positive molecular ion collisions (see, e.g. , Refs. 32-35). In the latter case, the energy range is less of a problem since electrons are used to probe the molecular ion. Moreover, merged-inclined-, and trapped-ion-beam configurations have helped to compensate for the low number density. Several experiments $32-35$ on $e+\mathrm{M}^{+}$have been performed over the last several years, usually by detecting dissociation products. Nevertheless, use of this source of information as a point of comparison for molecular photoionization processes has yet to see substantial progress.

\section{SHAPE RESONANCES IN h $\nu \div$ M AND e + M SYSTEMS}

Shape resonances are being observed as prominent features in the photoionization spectra and electron-scattering cross sections of a growing and diverse collection of molecules. On the surface, there is little connection, since the two phenomena involve different numbers of electrons and the collision velocities are such that all electrons are incorporated into the collision complex. Hence, we are comparing a neutral molecule versus a molecular negative-ion system. However, although the long-range part of the scattering potential is drastically different in the two cases, the inner part is not drastically different since it is dominated by the interactions with the nuclei and those electrons common to both systems. Thus, shape resonances which are localized in the molecular core substantially maintain their identity from one system to another, but are shifted in energy owing to the difference caused by the addition of an electron inside the molecule. This unifying property of shape resonances thus links together the two largest bodies of data for the molecular continuum - h $\nu+M$ and $e+M$-and although these resonances shift in energy in going from one class to another and manifest themselves in somewhat different ways, this link permits us to transfer information between the two. This can serve to help interpret new data and even to make predictions of new features to look for experimentally. Here we will review some of the evidence from experiment and from our recent calculations using the multiplescattering method, 36,37 concentrating on molecular photoionization, and elastic and vibrationally inelastic electron-molecule scattering.

Shape resonances are quasibound states supported by a potential barrier in the molecular potential seen by a continuum electron. The barrier is formed near the edge of the molecular charge distribution from a delicate balance between attractive (mainly Coulomb) and repulsive (mainly centrifugal) forces. Accordingiy, it usually effects only a single asymptotic partial wave in the final-state wave function. (Shape resonances that are essentially atomic in character can also occur in molecules for which a constituent atom can support a d-or f-wave shape resonance on its own.) Two uniquely molecular effects have been identified in recent studies (see, e.g., Ref. 37). First, the large spatial extent of the molec-

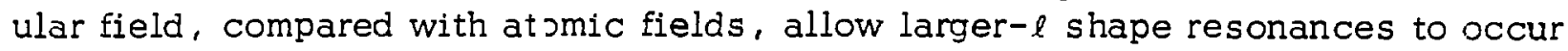
than observed in atomic sysiems. Second, the anisotropy of the molecular field can couple these resonant high- $\ell$ components to, e.g., $\mathrm{K}$-shell electrons in molecular photoionization. They generally stand out against a non-resonant background, especially in inner-shell photoionization, owing to their quasibound nature which produces a large amplitude inside the molecular core. (However, 
important exceptions to this statement exist, e.g., due to nuclear motion effects, strong non-resonant channels, etc.) Hence, shape resonances are convenient handles on continuum processes in molecules and are therefore very conducive to experimental and theoretical study.

As stated above, comparison of the spectroscopy of shape resonances in $M$ and $\mathrm{M}^{-}$systems is based on the premise that the potential inside the molecular core, which dominates the quasibound part of the shape resonance, is not drastically altered by the change in electron number. Hence, the $\ell$ character and shortrange charge distribution of a resonance in the two environments is substantially the same; however, the resonance energy is shifted to higher kinetic energies in the negative-ion system owing to the additional repulsive interaction accompanying the addition of an extra electron to the molecular field. Actually, this picture was surmised empirically from evidence contained in our survey calculations on $e+M$ and $h v+M$ and, in retrospect, from data. These observations can be summarized as follows: By and large, the systems $h v+M$ and $e+M$ display the same manifold of shape resonances, only those in the e+M system are shifted 5 to 15 $\mathrm{eV}$ to higher electron energy. In general, there is one shape resonance per symmetry for a subset of the symmetries available. The shift depends on the symmetry of the state, indicating, as one would expect, that the additional electron is not uniformly distributed. Finally, there is substantial proof that the $\ell$-character is preserved in this process, although interaction among altemative components in a scattering eigenchannel can alter the $\ell$ mixing present. Note that the localized nature of the shape resonance often permits rather accurate representation by virtual orbitals on LCAO calculations ${ }^{38}$ and by the stabilization method 39 in the negative ion system.

Several examples, including $\mathrm{N}_{2}, \mathrm{CO}, \mathrm{CO}_{2}, \mathrm{SF}_{6}$, etc.., will be given. Here we illustrate the case for $\mathrm{SF}_{6}$. In Fig. 2, the calculated elastic e-SF 6 scattering cross section 40 is shown. The total scattering cross section measured by Kennerly et al. 41 is included for comparison. The peaks in the $a_{1 g}, t_{1 u}, t_{2 g}$, and $e_{g}$ channels are shape :esonances. The agreement between theory and experiment, $l$ character of the resonances, and the absence of the $e_{g}$ feature in experiment are discussed elsewhere. 40 In Fig. 3 the sulfur $K$ - and L-shell photoionization spectra of $\mathrm{SF}_{6}$ (see Ref. 42 and references therein) are shown for comparison.

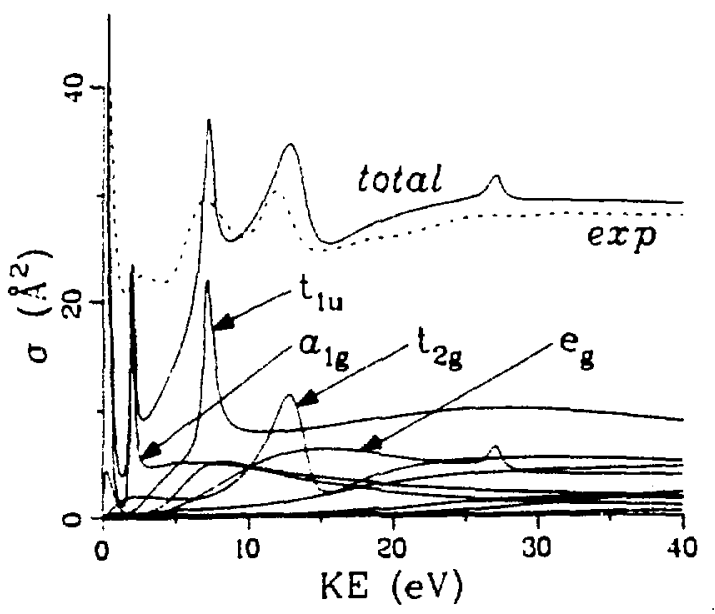

FIG. 2.--Comparison of e-SF 6 scattering cross sections. Solid curves are theoretical elastic cross sections. The dashed curve is the absolute total cross section measured by Kennerly et al. (Ref. 41). Only partial cross sections for resonant channels are labeled. Other channels are discussed in Rer. 40 .

The strong features, A, B, C, and D, are the same manifold of shape resonances, $\mathrm{a}_{1 \mathrm{~g}}, \mathrm{t}_{1 \mathrm{u}}, \mathrm{t}_{2 \mathrm{~g}}$, and $\mathrm{e}_{\mathrm{g}}$, shifted 5 to $15 \mathrm{eV}$ to lower kinetic energy. Note that the $\mathrm{a}_{1 \mathrm{~g}}$ and $\mathrm{t}_{1 \mathrm{u}}$ have slipped below threshold and are now highly-localized bound states. It is in the context of this comparison that we have, on occasion, referred 


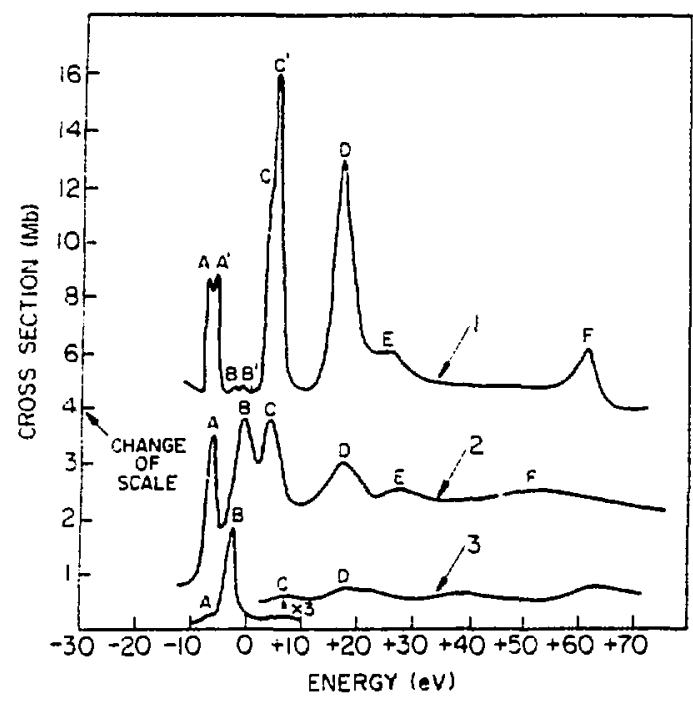

FIG. 3.--Sulfur L-shell (1), fluorine $\mathrm{K}-\mathrm{shell}(2)$, and sulfur $\mathrm{K}-\mathrm{shell}$ (3) photoabsorption spectra of $\mathrm{SF}_{6}$.

to these and analogous highly-localized discrete states as "discrete" shape resonances, although this is not to be taken in a literal sense. Figures 2 and 3 make an important point conceming the comparison between the manifestation of shape resonances in $h v+M$ and $e+M$ systems. The resonances are very prominent in the inner-shell photoionization spectra, whereas they do not dominate the elastic scattering cross section. This results mainly from the additional nonresonant scattering channels in electron scattering which are forbidden in photoionization by dipole selection rules. A similar effect generally occurs in going from innershell to valence-shell spectra, where again more final-state channels are frequently allowed, and the energy dependence of the dipole amplitude plays a greater role in the energy range of the resonances.

Another important, uniquely molecular effect is the interaction of shape resonances with vibrational motion. This topic was emphasized at the XIth ICPEAC ${ }^{43}$ last week, but will be touched on briefly here, as it does pertain to the connection between $h \nu+M$ and $e+M I$. Basically, since the shape resonance is localized in a spatial region of molecular dimensions by a centrifugal barrier which is a very sensitive function of internuclear separation $R$, the resonance position, width (lifetime), and profile are also sensitive functions of R. As shown elsewhere, 37, 43-49 this has four major consequences in the adiabatic nuclei approximation: Resonances calculated at the fixed equilibrium internuclear distance are smeared out, involving energy shifts and lowering and broadening of the resonance peak. This can even lead to the disappearance of a resonance in the total cross section. Second, excitation of vibration in e-M is greatly enhanced by shape resonances, even weak intermediate energy (10.to $40 \mathrm{eV}$ ) ones. This is important in observing weak resonances which would go unnoticed in the total scattering cross section. Third, in $h v+M$, this coupling of electronic and nuclear motion causes strongly non-Franck-Condon vibrational intensity distributions (mainly enhancement of the intensity of higher levels). In some cases, the shape resonance is hardly noticeable in the vibrationally unresolved partial cross section, but manifests itself in the vibrational intensity distribution. Fourth, the differential cross sections for $e-M$ and $h \nu+M$ are modified by coupling with nuclear motion, e.g., the $\beta$ parameters in $h \nu+M$ become strongly dependent on vibrational levels.

The final point of comparison involves angular-distribution studies. This is particularly meaningful in the case of shape resonances, since they usually have a very well-defined $\ell$ character. It is well known that the dominant $\ell$ component 
of a shape resonance in $e+M$ is manifested in the differential scattering cross section, unless it is strongly coupled with other $\ell$ components within an eigenchannel. Here we use the example of $\mathrm{N}_{2}$, for which $\pi_{\mathrm{g}}$ and $\sigma_{\mathrm{u}}$ shape resonances have been established 19,45 at 2.4 and $22 \mathrm{eV}$, respectively. The $\ell=2$ character of the strong $\pi_{\mathrm{g}}$ resonance is clearly shown 50 in Fig. 4 . In $h \nu+\mathrm{N}_{2}$, the $\pi_{\mathrm{g}}$ moves into the discrete and forms an intense autoionizing state (see, e.g. Refs. 51, 52). The $\sigma_{u}$ moves to $\sim 10 \mathrm{eV}$ and its angular distribution $52-54$ is easily observable. However, for random orientation, the photoelectron angular distribution is constrained 55 to the form

$$
\mathrm{d} \sigma / \mathrm{d} \Omega=(\sigma / 4 \pi)\left[1+\beta \mathrm{P}_{2}(\cos \theta)\right]
$$

so that, although the resonance is reflected in the energy dependence of $\beta, 52-54$ the $\ell$ character is suppressed. Only by fixing the orientation of the molecule, as in adsorption on a surface, does the $l$ character re-emerge. Figure 5 shows the angular distribution of photoelectrons ejected from the $\mathrm{K}$ shell of oriented $\mathrm{N}_{2}$ by light with polarization along the molecular axis, 37,56 so as to stimulate the resonant $\sigma_{u}$ channel. The predicted ${ }^{57} \ell=3$ character of the $\sigma_{u}$ resonance is clear.
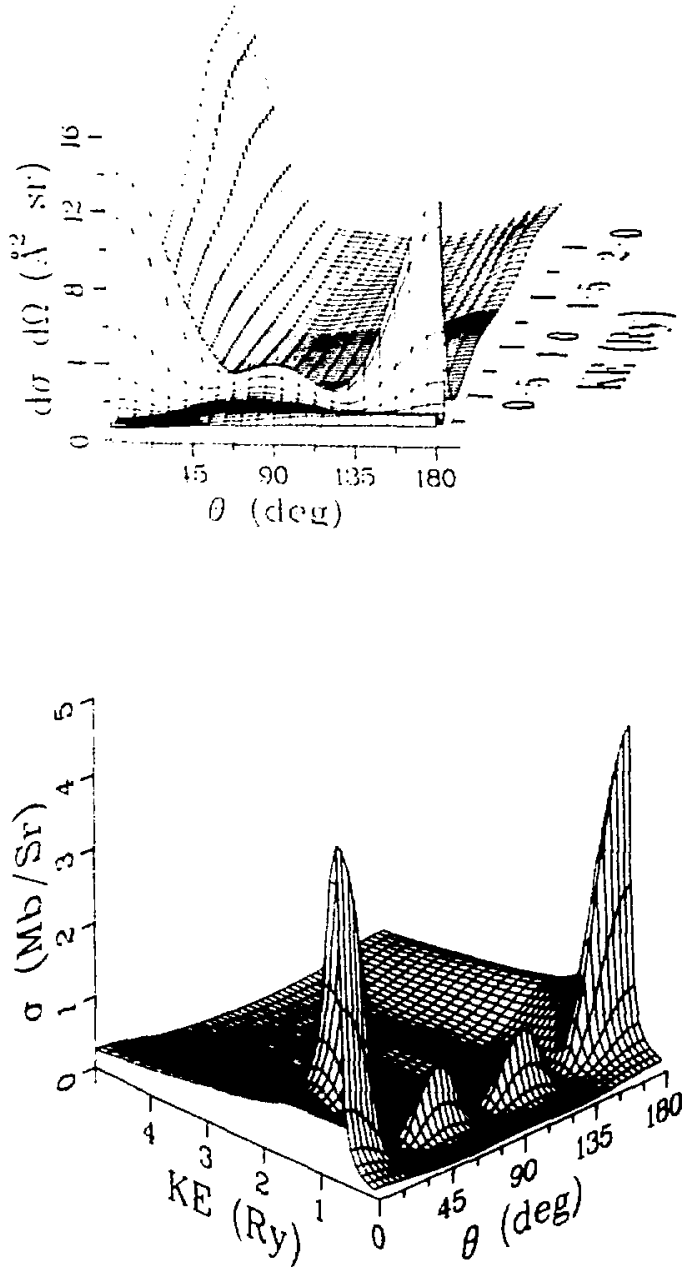

FIG. 4.--Differential scattering cross section for elastic e- $\mathrm{N}_{2}$ scattering, taken from Ref. 50 .
FIG. 5. --Photoelectron angular distribution for ejection of $\mathrm{K}-\mathrm{shell}$ electrons from oriented $\mathrm{N}_{2}$ by means of light linearly polarized along the molecular axis.

To summarize, we have emphasized the close connection between the manifold shape resonances in $e+M$ and $h v+M$ systems. An appreciation of this connection can permit the identification of features in $e+M$, given information on $h v+M$, and vice versa. At the next level, it will often be possible to predict unobserved effects in the same way, thus realizing the maximum benefit of a connection between electron-molecule scattering and molecular photoionization. 
We wish to thank Drs. Mitio Inokuti and David Spence for helpful conversations on several aspects of the present manuscript. We also wish to note that Section 2 borrows liberally from the ideas expressed by M. Inokuti in Ref. 6 .

\section{REFERENCES}

1. H. Bethe, Ann. Phys. (Leipzig) 5,325 (1930).

2. H. Bethe, Handbuch der Physik, Eds., H. Geiger and K. Scheel (Springer, Berlin, 1933), Vol. 24/1, p. 273

3. M. Inokuti, Rev. Mod. Phys. 43, 297 (1971).

4. M. Inokuti, Invited Papers and Progress Reports, VIIth ICPEAC, Amsterdam, 1971. Eds., T. R. Govers and F. J. de Heer (North-Holland, Amsterdam, 1972), p. 327 .

5. E. N. Lassettre and A. Skerbele, Methods of Experimental Physics, Vol. 3 , Molecular Physics, Part B, Ed., D. Williams (Academic Press, New York, 1974), Chapter 7.2, p. 868 .

6. M. Inokuti, Photoionization and Other Probes of Many-Electron Interactions, Ed. , F. Wuilleumier (Plenum, New York, 1976), p. 165.

7. M. J. van der Wiel, ibid., pp. 187 and 199.

8. M. J. van der Wiel and C. E. Brion, J. Electron Spectrosc. 1, 439 (1972).

9. C. E. Brion, A. Fiammett, G. R. Wight, and M. J van der Wiel, J. Electron Spectrosc. 12, 323 (1977).

10. A. P. Hitchcock, C. E. Brion, and J. M. van der Wiel, J. Phys. B 11,3245 (1978).

11. K. H. Tan, C. E. Brion, Ph. E. van der Leeuw, and M. J. van der Wiel, Chem. Phys. 29, 299 (1978).

12. M. Tronc, G. C. King, R. C. Bradford, and F. H. Read, J. Phys. B 9 , L555 (1976).

13. M. Tronc, G. C. King, and F. H. Read, J. Phys. B 12, 137 (1979).

14. Y.-K. Kim, Radiat. Res. 61, 21 (1975); 64, 96 (1975); 64, 205 (1975).

15. U. Fano, J. Opt. Soc. Am. 65, 979 (1975).

16. C. M. Lee, Phys. Rev. A 16,109 (1977).

17. D. Dill and Ch. Jungen, J. Phys. Chem., in press.

18. M. Inokuti, Photoionization and Other Probes of Many-Electron Interactions, Ed., F. Wuilleumier (Plenum, New York, 1976), p. 431.

19. G. J. Schulz, Rev. Mod. Phys. 45, 422 (1973).

20. M. W. Siegel, R. J. Celotta, J. L. Hall, J. Levine, and R. A. Bennett, Phys. Rev. A $\underline{6}, 607$ (1972).

21. R. J. Celotta, R. A. Bennett, J. L. Hall, M. W. Siegel, and J. Levine, Phys. Rev. A $\underline{6}, 631$ (1972).

22. H. Hotop, T. A. Patterson, and W. C. Lineberger, J. Chem. Phys. 60 , 1806 (1974).

23. E. Herbst, T. A. Patterson, and W. C. Lineberger, J. Chem. Phys. 61, 1300 (1974).

24. A. Kasdan, E. Herbst, and W. C. Lineberger, Chem. Phys. Lett. 31, 78 (1975).

25. P. F. Zittel and W. C. Lineberger, J. Chem. Phys. 65, 1932 (1976).

26. P. C. Engelking and W. C. Lineberger, J. Chem. Phys. 66, 5054 (1977).

27. P.A.M. Gram, J. C. Pratt, M. A. Yates-Williams, H. C. Bryant, J. Donahue, H. Sharifian, and H. Tootoonchi, Phys - Rev. Lett. $\underline{40}, 107$ (1978).

28. J. Macek and P. G. Burke, Proc. Phys. Soc. London 92, 351 (1967);

J. Macek, Proc. Phys. Soc. London 92, 365 (1967).

29. C. D. Lin, Phys. Rev. Lett. 35,1150 (1975). 
30. J. T. Broad and W. P. Reinhardt, Phys. Rev. A 14, 2159 (1976).

31. G. J. Schulz, Rev. Mod. Phys. 45,378 (1973).

32. K. T. Dolder and B. Peart, Reports on Progress in Physics $39,693(1976)$.

33. R. A. Heppner, F. L. Wall, W. T. Armstrong, and G. H. Dunn, Phys. Rev. A 13,1000 (1976).

34. D. Mathur, J. B. Hasted, and S. U. Khan, J. Phys. B 12, 2043 (1979).

35. J. W. MoGowan, R. Candano, and J. Keyser, Phys. Rev. Lett. 36, 1447 (1976).

36. D. Dill and J. L. Dehmer, J. Chem. Phys. 61, 692 (1974).

37. J. L. Dehmer and D. Dill, Electron-and Photon-Molecule Collisions, V. McKoy, T. Rescigno, and B. Schneider, Eds., (Plenum Press, New York, 1979) in press.

38. F. A. Gianturco, C. Guidotti, and V. Lamanna, J. Chem. Phys. 57, 840 (1972).

39. H. S. Taylor, G. V. Nagarov, and A. Golebiewski, J. Chem. Phys. 45, $2873(1966)$.

40. J. L. Dehmer, J. Siegel, and D. Dill, J. Chem. Phys. 69, 5205 (1978).

41. R. E. Kennerly, R. A. Bonham, and M. McMillan, J. Chem. Phys. 70, 2039 (1979).

42. J. L. Dehmer, J. Chem. Phys. $\underline{56}, 4496$ (1972), and references therein.

43. J. L. Dehmer and D. Dill, Progress Report, XI ICPEAC.

44. M. G. Lynch, D. Dill, I. Siegel, and J. L. Dehmer, J. Chem. Phys., in press.

45. J. L. Dehmer, J. Siegel, J. Welch, and D. Dill, Phys. Rev. A, to be published.

46. D. Dill, J. Welch, J. L. Dehmer, and J. Siegel, to be published.

47. T. I. Dehmer, D. Dill, and S. Wallace, submitted to Phys. Rev. Letters.

48. R. Stockbauer, B. E. Cole, D. L. Ederer, J. B. West, A. C. Parr, J. L. Dehmer, Phys. Rev. Letters, in press.

49. M. Tronc, R. Azria, and R. Paineau, J. de Physique Lettres, in press.

50. J. Siegel, D. Dill, and J. L. Dehmer, Phys. Rev. A 17, 2106 (1978).

51. G. R. Wight, C. E. Brion, and M. J. van der Wiel, J. Electron Spectrosc. 1,457 (1973).

52. J. L. Dehmer and D. Dill, J. Chem. Phys. 65, 5327 (1976).

53. G. V. Marr, J. M. Morton, R. M. Holmes, and D. G. McCoy, J. Phys. B $12,43(1979)$.

54. S. Wallace, D. Dill, and J. L. Dehmer, J. Phys. B, in press.

55. C. N. Yang, Phys. Rev. 74,764 (1948).

56. D. Dill, J. Siegel, and J. I. Dehmer, J. Chem. Phys. 65, 3158 (1976).

57. J. L. Dehmer and D. Dill, Phys. Rev. Lett. 35,213 (1975). 